\title{
Copper tolerance and distribution of epibiotic bacteria associated with giant kelp Macrocystis pyrifera in southern California
}

\author{
Julia Busch ${ }^{1,2}$ - Juliana Ribeiro Nascimento ${ }^{3}$ Ana Carolina Rubem Magalhães ${ }^{4}$. \\ Bas E. Dutilh ${ }^{5,6} \cdot$ Elizabeth Dinsdale $^{1}$
}

Accepted: 6 April 2015/Published online: 18 April 2015

(C) The Author(s) 2015. This article is published with open access at Springerlink.com

\begin{abstract}
Kelp forests in southern California are important ecosystems that provide habitat and nutrition to a multitude of species. Macrocystis pyrifera and other brown algae that dominate kelp forests, produce negatively charged polysaccharides on the cell surface, which have the ability to accumulate transition metals such as copper. Kelp forests near areas with high levels of boating and other industrial activities are exposed to increased amounts of these metals, leading to increased concentrations on the algal surface. The increased concentration of transition metals creates a harsh environment for colonizing microbes altering community structure. The impact of altered bacterial populations in the kelp forest have unknown consequences that could be harmful to the health of the ecosystem. In this study we describe the community of microorganisms associated with $M$. pyrifera, using a culture based approach, and their increasing tolerance to the
\end{abstract}

Elizabeth Dinsdale

elizabeth_dinsdale@hotmail.com

1 Biology Department, San Diego State University, San Diego, California, USA

2 Present Address: Scripps Institution of Oceanography, University of California, San Diego, USA

3 Marine Biology Institute-CCS, Universidade Federal do Rio de Janeiro, Rio de Janeiro, Brazil

4 Biology Department, Federal Fluminense University, Niterói, Rio de Janeiro, Brazil

5 Computer Science Department, San Diego State University, California, USA

6 Centre for Molecular and Biomolecular Informatics, CMBI, Nijmegen Centre for Molecular Life Sciences, Radboud University Nijmegen Medical Centre, Geert Crooteplein 28, 6526 GA Nijmegen, The Netherlands transition metal, copper, across a gradient of human activity in southern California. The results support the hypothesis that $M$. pyrifera forms a distinct marine microhabitat and selects for species of bacteria that are rarer in the water column, and that copper-resistant isolates are selected for in locations with elevated exposure to transition metals associated with human activity.

Keywords Copper tolerance $\cdot$ Bacterial isolate $\cdot$ Kelp forest

\section{Introduction}

Kelp forests are one of the most widespread and productive ecosystems. Found in shallow temperate coastal waters as well as deeper tropical waters, kelp forests are dominated by brown algae from the genus Laminaria (Santelices 2007), such as Macrocystis pyrifera (giant kelp) and their high productivity provide habitats and nutrients to an entire ecosystem (Egan et al. 2008; Goecke et al. 2010). The nutrient-rich surface of the macroalgae provides a desirable location for epibiotic colonization resulting in higher abundance and diversity of microbial populations (Egan et al. 2008; Blight and Thompson 2008; Armstrong et al. 2001; Lachnit et al. 2011). Kelp produces a matrix of acidic polysaccharides that have a high binding affinity for transition metals such as copper (Davis et al. 2003; Andrade et al. 2004, 2010). While the algal surface is desirable for colonization, in locations where the amount of transition metals is increased by human activity, the bioaccumulation of metals by the kelp may create a stressful environment for bacteria.

Urbanization, industrialization and boating activity can increase the levels of transition metals entering the ocean. 
In particular, copper-based paint used to prevent marine fouling on boat hulls contributes to elevated levels of transition metals in marinas and bays around the world (Dobalian and Arias 2005; Biggs and D'Anna 2012; Schiff et al. 2006). In 2004 the average copper level in San Diego Bay was 8 ppb (Johnson and Gonzalez 2005), a 36-fold increase of the natural level of $0.225 \mathrm{ppb}$ in ocean water (Blossom 2007). While copper is an essential micronutrient, in high levels it is lethal. The distribution of marine cyanobacteria cells is adversely affected by copper exposure and strains from populated coastal areas are more tolerant to elevated copper levels than those from the open ocean (Stuart et al. 2009; Moffett et al. 2012). However, little research has been conducted on the microbes associated with the kelp forest and the effects of transition metal accumulation. In southern California, the combination of high boating activity and the close proximity of kelp forests to marinas may increase the bioaccumulation of transition metals. To examine whether there was an effect of bioaccumulation of transition metals on microbial populations, we isolated bacteria from the kelp surface and surrounding water at three locations in southern California and described their taxonomic distribution and effect of copper on their growth.

\section{Materials and methods}

Bacteria were cultured from blades of $M$. pyrifera and surface seawater from three different kelp forest locations in southern California: The Point Loma kelp forest $\left(32^{\circ} 39^{\prime} 59.56^{\prime \prime} \mathrm{N}, 117^{\circ} 14^{\prime} 50.62^{\prime \prime} \mathrm{W}\right)$ close to the port of San Diego, La Jolla kelp forest $\left(32^{\circ} 50^{\prime} 3.71^{\prime \prime} \mathrm{N}, 117^{\circ} 16^{\prime} 54.57^{\prime \prime} \mathrm{W}\right)$ located adjacent to an urban area but with minimal boating activity, and Santa Catalina Island $\left(33^{\circ} 27^{\prime} 1.89^{\prime \prime} \mathrm{N}\right.$, $118^{\circ} 29^{\prime} 12.32^{\prime \prime} \mathrm{W}$ ) located adjacent a marine protected area approximately $70 \mathrm{~km}$ from the mainland (Fig. 1). Bacteria associated with the surface of the algae were obtained by washing the blade in $0.02 \mu \mathrm{m}$-filtered seawater to remove loosely associated bacteria. After washing, the kelp was wiped across the media plates transferring the viscous surface mucus and associated microbes onto the agar. Bacteria closely associated with the kelp blade tissue were isolated using a technique adapted from Burke et al. (2009) in which kelp surface-associated bacteria were eliminated before plating. The kelp blade pieces were washed in a $1 \%$ bleach solution with gentle agitation for $30 \mathrm{~s}$ followed by two washes in $0.02 \mu \mathrm{m}$ filtered seawater and the blade pieces were applied directly onto the agar. Additional bacterial isolates were obtained by crushing the kelp blade using a sterile mortar and pestle and a $100 \mu \mathrm{l}$ sample was pipetted onto the media. Bacteria from the water column were obtained from around the kelp blades and $100 \mu$ plated. The media used included TCBS (Neogen Cat. No. 7210A) and
Marine Broth (Difco Laboratories Cat. No. 2013-10-31) with $15 \mathrm{~g} / \mathrm{l}$ agar added. Bacteria were cultured at room temperature and streaked until purification. A total of 600 bacterial isolates were grown and a random set (98) were selected for RFLP analysis and copper tolerance assays.

Bacterial DNA was extracted using nuclei lysis buffer and protein precipitate solution. 16S PCR amplification was performed in which the DNA was denatured for $5 \mathrm{~min}$ at $94{ }^{\circ} \mathrm{C}$ and amplified through 30 cycles of $94{ }^{\circ} \mathrm{C}$ for $1 \mathrm{~min}$, $65{ }^{\circ} \mathrm{C}$ for $1 \mathrm{~min}$ with $0.5^{\circ} \mathrm{C}$ touchdown, $72{ }^{\circ} \mathrm{C}$ for $3 \mathrm{~min}$, with a final extension of $72{ }^{\circ} \mathrm{C}$ for $10 \mathrm{~min}$. The amplified $16 \mathrm{~S}$ rDNA was detected by electrophoresis on a $1 \%$ agarose gel with $0.5 \mu \mathrm{g} / \mathrm{ml}$ ethidium bromide in $1 \times$ TBE buffer.

To investigate the types of bacteria that were associated with either kelp or the water column at the three locations we conducted a restriction fragment length polymorphism (RFLP) analysis. The $16 \mathrm{~S}$ sequences of 98 randomly selected bacteria were purified and digested using three restriction endonucleases: ScaI, HaeIII, and MspI. The digested 16S rDNA was run on a $2 \%$ agarose gel followed by staining in ethidium bromide with a final concentration of $0.5 \mu \mathrm{g} / \mathrm{ml}$. The $16 \mathrm{~S}$ rDNA PCR product of one isolate from each RFLP group was sequenced using Sanger sequencing. Two sequences were sequenced from Pattern A to investigate whether the restriction pattern were unique to a species. The sequences were aligned with MUSCLE (Edgar 2004) and a maximum likelihood phylogenetic tree was created using RAxML (Stamatakis 2014) with standard settings and 100 bootstrap repetitions. Reference sequences for the phylogenetic analysis where chosen from NCBI after the alignment to identify the type strain of the closes matches to our $16 \mathrm{~S}$ sequences. The type strains used were of Alteromonas addita, Photobacterium phosphoreum, Pseudoalteromonas arctica, Pseudoalteromonas citrea, Pseudoalteromonas espejiana, Psychroserpens burtonensis, Ruegeria mobilis, Shewanella fidelia, Vibrio cyclitrophicus, Vibrio mediterranei, Vibrio parahaemolyticus, Vibrio splendidus, and Vibrio wodanis (accession numbers: AY682202, PHR16SRD4, DQ787199, X82137, X82143, PBU62913, AB255401, AF420312, AM162656, X74710, AF388386, AJ515230, AJ132227 respectively).

The accumulation of copper in kelp was compared for Pt. Loma, near the highest human occupation and Catalina near the lowest level of human occupation in this study. The amount of copper in M. pyrifera blades were quantified using an acid digestion method modified from Warnau et al. (1995). The digested samples were analyzed on a Perkin-Elmer DV 4300 inductively coupled plasmid atomic emissions spectrometer (ICP-AES) and conversion calculations were performed to determine the for $\mathrm{Cu}$ concentrations. Differences between the copper concentrations at Catalina and Pt. Loma were compared using a student $t$ test. Water quality measurements, including temperature, salinity, $\mathrm{pH}$, dissolved oxygen, and chlorophyll-a were 


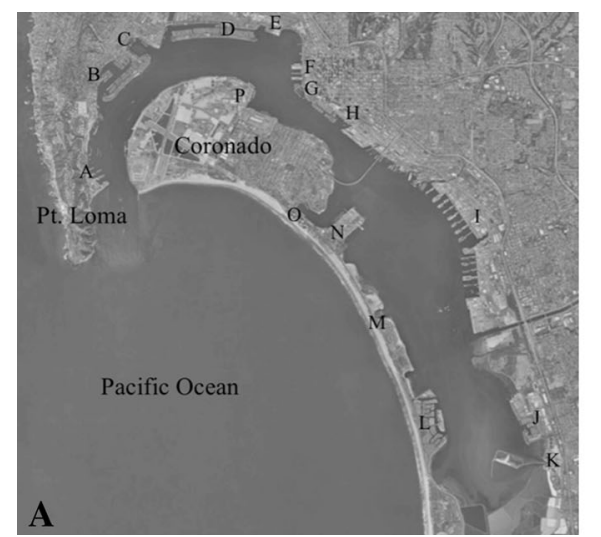

Fig. 1 a A map of the San Diego Bay labeled with the areas with high boating activity that contribute to the copper load in the water. $a$ Navy Submarine Base; $b$ Shelter Island; $c$ Fisherman's Landing; $d$ Harbor Island; $e$ US Coast Guard; $f$ B St. Cruise Ship Terminal, Broadway Pier, USS Midway; $g$ Tuna Basin; $h$ Embarcadero Marina; $i$ US Naval Station; $j$ Chula Vista Yacht Club; $k$ South Bay Power

taken at surface $(\sim 0.5 \mathrm{~m})$ and benthos $(\sim 12 \mathrm{~m})$ for both the Pt. Loma and Catalina Island kelp forest collection sites with a MANTA-2 multiprobe (Measurement Specialties, VA, USA). A two sample two sided $t$ test for each of the water quality measurements was use to compare the mean from three replicate measurements.

Growth inhibition assays were conducted in order to identify whether the bacteria isolated from different kelp forests had varying tolerance to copper. The median inhibitory concentration $\left(\mathrm{IC}_{50}\right)$ of a bacterial isolate was defined as the lowest concentration of $\mathrm{Cu}$ at which growth was half the control value. $\mathrm{IC}_{50}$ assays were performed on ten bacterial isolates from Pt. Loma, ten bacterial isolates from La Jolla, and eight bacterial isolates from Catalina. A stock copper solution of $\mathrm{CuSO}_{4} \cdot 5 \mathrm{H}_{2} \mathrm{O}$ in deionized water was prepared and sterilized. Bacterial isolates were grown from 1:1000 inoculations of overnight cultures in sterile Zobell Marine Broth 2216 ( Difco $^{\mathrm{TM}}$ ) with ranging concentrations of copper. Five replicates of each copper concentration were incubated for $18-20 \mathrm{~h}$ at room temperature. Optical density was measured at $550 \mathrm{~nm}$ to compare bacterial growth inhibition. To explore the relationship of kelp-associated and seawater isolates and growth inhibition by copper, a one-way ANOVA test was conducted, with the effect of geographic location and sample type (i.e., kelp or seawater) being tested.

\section{Results}

To investigate whether sample type (i.e., kelp tissue or water) or geographic location affected bacterial distribution, a random selection of 98 isolates was classified and 18 different RFLP patterns (labeled A through R) were

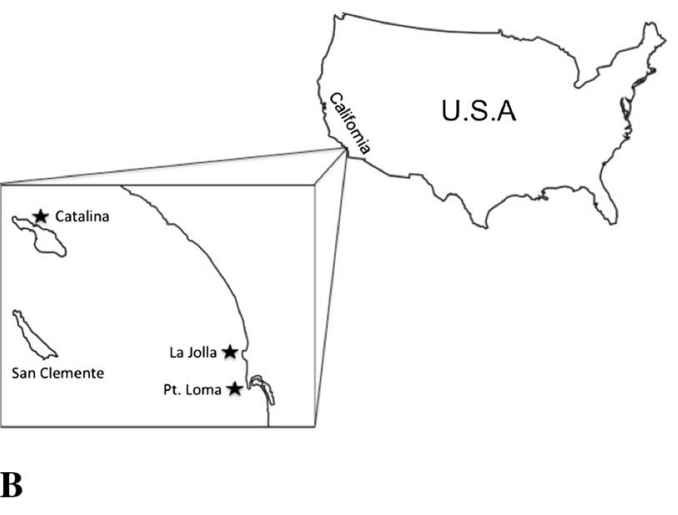

Plant; $l$ Coronado Cays; $m$ Fiddler's Cove; $n$ Naval Amphibious Base Coronado; $o$ Glorietta Bay; $p$ Naval Air Station North Island. b Sampling locations off the coast of southern California. La Jolla $\left(32^{\circ} 50^{\prime} 3.71^{\prime \prime} \mathrm{N}, \quad 117^{\circ} 16^{\prime} 54.57^{\prime \prime} \mathrm{W}\right) ; \quad$ Pt. Loma $\left(32^{\circ} 39^{\prime} 59.56^{\prime \prime} \mathrm{N}\right.$, $\left.117^{\circ} 14^{\prime} 50.62^{\prime \prime} \mathrm{W}\right)$; Catalina $\left(33^{\circ} 27^{\prime} 1.89^{\prime \prime} \mathrm{N}, 118^{\circ} 29^{\prime} 12.32^{\prime \prime} \mathrm{W}\right)$

identified (Table 1). Of the 98 isolates, 57 were Vibrio species and were found in both kelp and water from all three locations (Table 1,2). Bacteria with RFLP Pattern A-most similar to $V$. splendidus were the most abundant and widespread, while Pattern B-most similar to $V$. $c y$ clitrophicus was found across all three locations but not all sample types (Table 1). In addition to geographical differences, eight bacterial species most similar to Ruegeria sp., three Pseudoalteromonas spp., Alteromonas sp., two additional Vibrio spp., and Psychroserpens sp. were specific to kelp ( $n=18$ isolates), and two groups of bacteria were found exclusively in water ( $n=7$ isolates) including species similar to $V$. wodanis and $P$. phosphoreum.

Isolates from geographic locations and sample types were distributed evenly throughout the phylogenetic tree (Fig. 2). The Vibrio and Pseudoalteromonas species clustered into two separated groups and bacteria isolates that had the RLFP Pattern D were not similar to anything in the database and appeared as an outlier in the phylogenetic analysis, suggesting it is a new species based on the $16 \mathrm{~S}$ rDNA sequence (Fig. 2).

The concentration of copper found in the kelp from Pt. Loma was greater with a mean of $6.21( \pm 0.19) \mathrm{ppm}$ compared with the kelp from Catalina which had a mean of $4.33( \pm 0.08) \mathrm{ppm}\left(\boldsymbol{T}_{d f=4}=10.334, p<0.001\right)$. No data was collected from La Jolla. Water quality measurements indicate that the Pt. Loma and Catalina Island kelp forests were similar at surface depths for dissolved oxygen levels $(p=0.345)$ and salinity $(p=0.107)$, and similar at the benthos for chlorophyll-a $(p=0.891)$. In contrast, temperature was higher at the surface in Pt. Loma compared to Catalina $\left[\mathrm{x}_{\text {PtLoma }}=16.807( \pm 0.039){ }^{\circ} \mathrm{C}, \overline{\mathrm{x}}_{\text {Catalina }}=16.593\right.$ $\left.( \pm 0.009){ }^{\circ} \mathrm{C}\right](p=0.027)$, yet lower in the benthos of Pt. 
Table 1 Sizes of RFLP fragments obtained from PCR amplified 16S rDNA of marine bacterial isolates

\begin{tabular}{|c|c|c|c|c|c|}
\hline Stock number & Location & ScaI & HaeIII & MspI & Restriction pattern \\
\hline 156 & Catalina kelp & 1100,475 & $310,290,210,190,150$ & $550,520,125$ & A \\
\hline 157 & Catalina kelp & 1100,475 & $310,290,210,190,150$ & $550,520,125$ & A \\
\hline 260 & Catalina kelp & 1100,475 & $310,290,210,190,150$ & $550,520,125$ & A \\
\hline 330 & Catalina kelp & 1100,475 & $310,290,210,190,150$ & $550,520,125$ & A \\
\hline 131 & Catalina water & 1100,475 & $310,290,210,190,150$ & $550,520,125$ & A \\
\hline 147 & Catalina water & 1100,475 & $310,290,210,190,150$ & $550,520,125$ & A \\
\hline 149 & Catalina water & 1100,475 & $310,290,210,190,150$ & $550,520,125$ & A \\
\hline 189 & Catalina water & 1100,475 & $310,290,210,190,150$ & $550,520,125$ & A \\
\hline 3 & La Jolla kelp & 1100,475 & $310,290,210,190,150$ & $550,520,125$ & A \\
\hline 4 & La Jolla kelp & 1100,475 & $310,290,210,190,150$ & $550,520,125$ & A \\
\hline 5 & La Jolla kelp & 1100,475 & $310,290,210,190,150$ & $550,520,125$ & A \\
\hline 592 & La Jolla kelp & 1100,475 & $310,290,210,190,150$ & $550,520,125$ & A \\
\hline 593 & La Jolla kelp & 1100,475 & $310,290,210,190,150$ & $550,520,125$ & A \\
\hline 594 & La Jolla kelp & 1100,475 & $310,290,210,190,150$ & $550,520,125$ & A \\
\hline 595 & La Jolla kelp & 1100,475 & $310,290,210,190,150$ & $550,520,125$ & A \\
\hline 596 & La Jolla kelp & 1100,475 & $310,290,210,190,150$ & $550,520,125$ & A \\
\hline 598 & La Jolla kelp & 1100,475 & $310,290,210,190,150$ & $550,520,125$ & A \\
\hline 599 & La Jolla kelp & 1100,475 & $310,290,210,190,150$ & $550,520,125$ & A \\
\hline 582 & La Jolla water & 1100,475 & $310,290,210,190,150$ & $550,520,125$ & A \\
\hline 584 & La Jolla water & 1100,475 & $310,290,210,190,150$ & $550,520,125$ & A \\
\hline 585 & La Jolla water & 1100,475 & $310,290,210,190,150$ & $550,520,125$ & A \\
\hline 586 & La Jolla water & 1100,475 & $310,290,210,190,150$ & $550,520,125$ & A \\
\hline 590 & La Jolla water & 1100,475 & $310,290,210,190,150$ & $550,520,125$ & A \\
\hline 591 & La Jolla water & 1100,475 & $310,290,210,190,150$ & $550,520,125$ & A \\
\hline 642 & La Jolla water & 1100,475 & $310,290,210,190,150$ & $550,520,125$ & A \\
\hline 400 & Pt. Loma water & 1100,475 & $310,290,210,190,150$ & $550,520,125$ & A \\
\hline 401 & Pt. Loma water & 1100,475 & $310,290,210,190,150$ & $550,520,125$ & A \\
\hline 403 & Pt. Loma water & 1100,475 & $310,290,210,190,150$ & $550,520,125$ & A \\
\hline 404 & Pt. Loma water & 1100,475 & $310,290,210,190,150$ & $550,520,125$ & A \\
\hline 406 & Pt. Loma water & 1100,475 & $310,290,210,190,150$ & $550,520,125$ & A \\
\hline 407 & Pt. Loma water & 1100,475 & $310,290,210,190,150$ & $550,520,125$ & A \\
\hline 252 & Catalina water & 1100,475 & $310,290,210,190,150$ & 550,520 & B \\
\hline 411 & Catalina water & 1100,475 & $310,290,210,190,150$ & 550,520 & B \\
\hline 653 & La Jolla kelp & 1100,475 & $310,290,210,190,150$ & 550,520 & B \\
\hline 643 & La Jolla water & 1100,475 & $310,290,210,190,150$ & 550,520 & B \\
\hline 478 & Pt. Loma kelp & 1100,475 & $310,290,210,190,150$ & 550,520 & B \\
\hline 479 & Pt. Loma kelp & 1100,475 & $310,290,210,190,150$ & 550,520 & B \\
\hline 480 & Pt. Loma kelp & 1100,475 & $310,290,210,190,150$ & 550,520 & B \\
\hline 482 & Pt. Loma kelp & 1100,475 & $310,290,210,190,150$ & 550,520 & B \\
\hline 501 & Pt. Loma kelp & 1100,475 & $310,290,210,190,150$ & 550,520 & B \\
\hline 129 & Catalina kelp & Uncut & $310,290,210,190,150$ & 550,520 & $\mathrm{C}$ \\
\hline 136 & Catalina kelp & Uncut & $310,290,210,190,150$ & 550,520 & $\mathrm{C}$ \\
\hline 517 & Catalina kelp & Uncut & $310,290,210,190,150$ & 550,520 & $\mathrm{C}$ \\
\hline 120 & Catalina water & Uncut & $310,290,210,190,150$ & 550,520 & $\mathrm{C}$ \\
\hline 121 & Catalina water & Uncut & $310,290,210,190,150$ & 550,520 & $\mathrm{C}$ \\
\hline 122 & Catalina water & Uncut & $310,290,210,190,150$ & 550,520 & $\mathrm{C}$ \\
\hline 137 & Catalina water & Uncut & $310,290,210,190,150$ & 550,520 & $\mathrm{C}$ \\
\hline 515 & Catalina water & Uncut & $310,290,210,190,150$ & 550,520 & $\mathrm{C}$ \\
\hline
\end{tabular}


Table 1 continued

\begin{tabular}{|c|c|c|c|c|c|}
\hline Stock number & Location & ScaI & HaeIII & MspI & Restriction pattern \\
\hline 635 & Catalina water & Uncut & $310,290,210,190,150$ & 550,520 & $\mathrm{C}$ \\
\hline 522 & Pt. Loma kelp & Uncut & $500,290,190,125$ & $600,475,275,150$ & $\mathrm{D}$ \\
\hline 523 & Pt. Loma kelp & Uncut & $500,290,190,125$ & $600,475,275,150$ & $\mathrm{D}$ \\
\hline 524 & Pt. Loma kelp & Uncut & $500,290,190,125$ & $600,475,275,150$ & $\mathrm{D}$ \\
\hline 525 & Pt. Loma kelp & Uncut & $500,290,190,125$ & $600,475,275,150$ & $\mathrm{D}$ \\
\hline 526 & Pt. Loma kelp & Uncut & $500,290,190,125$ & $600,475,275,150$ & $\mathrm{D}$ \\
\hline 527 & Pt. Loma kelp & Uncut & $500,290,190,125$ & $600,475,275,150$ & $\mathrm{D}$ \\
\hline 575 & Pt. Loma kelp & Uncut & $500,290,190,125$ & $600,475,275,150$ & $\mathrm{D}$ \\
\hline 576 & Pt. Loma kelp & Uncut & $500,290,190,125$ & $600,475,275,150$ & $\mathrm{D}$ \\
\hline 583 & La Jolla water & Uncut & $500,290,190,125,100$ & $500,375,190,125$ & $\mathrm{E}$ \\
\hline 644 & La Jolla water & Uncut & $500,290,190,125,100$ & $500,375,190,125$ & $\mathrm{E}$ \\
\hline 645 & La Jolla water & Uncut & $500,290,190,125,100$ & $500,375,190,125$ & $\mathrm{E}$ \\
\hline 646 & La Jolla water & Uncut & $500,290,190,125,100$ & $500,375,190,125$ & $\mathrm{E}$ \\
\hline 483 & Pt. Loma kelp & Uncut & $500,290,190,125,100$ & $500,375,190,125$ & $\mathrm{E}$ \\
\hline 484 & Pt. Loma kelp & Uncut & $500,290,190,125,100$ & $500,375,190,125$ & $\mathrm{E}$ \\
\hline 485 & Pt. Loma kelp & Uncut & $500,290,190,125,100$ & $500,375,190,125$ & $\mathrm{E}$ \\
\hline 521 & Pt. Loma kelp & Uncut & $500,290,190,125,100$ & $500,375,190,125$ & $\mathrm{E}$ \\
\hline 588 & La Jolla kelp & 1100,475 & $500,290,190,125$ & 550,520 & $\mathrm{~F}$ \\
\hline 654 & La Jolla kelp & 1100,475 & $500,290,190,125$ & 550,520 & $\mathrm{~F}$ \\
\hline 657 & La Jolla kelp & 1100,475 & $500,290,190,125$ & 550,520 & $\mathrm{~F}$ \\
\hline 658 & La Jolla kelp & 1100,475 & $500,290,190,125$ & 550,520 & $\mathrm{~F}$ \\
\hline 659 & La Jolla kelp & 1100,475 & $500,290,190,125$ & 550,520 & $\mathrm{~F}$ \\
\hline 581 & La Jolla water & 1100,475 & $500,290,190,125$ & 550,520 & $\mathrm{~F}$ \\
\hline 132 & Catalina water & 1100,475 & $310,290,210,190,150$ & 510,480 & G \\
\hline 187 & Catalina water & 1100,475 & $310,290,210,190,150$ & 510,480 & G \\
\hline 253 & Catalina water & 1100,475 & $310,290,210,190,150$ & 510,480 & G \\
\hline 412 & Catalina water & 1100,475 & $310,290,210,190,150$ & 510,480 & G \\
\hline 639 & La Jolla water & 1100,475 & $310,290,210,190,150$ & 510,480 & G \\
\hline 127 & Catalina kelp & 1100,475 & $310,290,250,210,190,150$ & 550,520 & $\mathrm{H}$ \\
\hline 133 & Catalina kelp & 1100,475 & $310,290,250,210,190,150$ & 550,520 & $\mathrm{H}$ \\
\hline 134 & Catalina kelp & 1100,475 & $310,290,250,210,190,150$ & 550,520 & $\mathrm{H}$ \\
\hline 135 & Catalina kelp & 1100,475 & $310,290,250,210,190,150$ & 550,520 & $\mathrm{H}$ \\
\hline 641 & La Jolla water & 1100,475 & $310,290,250,210,190,150$ & 550,520 & $\mathrm{H}$ \\
\hline 329 & Catalina kelp & Uncut & $310,290,210,190,150$ & $510,480,125$ & I \\
\hline 416 & Catalina kelp & Uncut & $310,290,210,190,150$ & $510,480,125$ & I \\
\hline 139 & Catalina water & Uncut & $310,290,210,190,150$ & $510,480,125$ & I \\
\hline 248 & Catalina kelp & 1100,475 & $500,290,210,190,125$ & $510,375,180$ & $\mathrm{~J}$ \\
\hline 647 & La Jolla water & 1100,475 & $500,290,210,190,125$ & $510,375,180$ & $\mathrm{~J}$ \\
\hline 587 & La Jolla kelp & $850,475,225$ & $500,290,190,125$ & 550,520 & $\mathrm{~K}$ \\
\hline 589 & La Jolla kelp & $850,475,225$ & $500,290,190,125$ & 550,520 & $\mathrm{~K}$ \\
\hline 656 & La Jolla kelp & Uncut & $310,290,190,150$ & 550,520 & $\mathrm{~L}$ \\
\hline 486 & Pt. Loma kelp & Uncut & $310,290,190,150$ & 550,520 & $\mathrm{~L}$ \\
\hline 142 & Catalina kelp & Uncut & $500,290,190,125$ & $510,375,180$ & M \\
\hline 143 & Catalina kelp & Uncut & $500,290,190,125$ & $510,375,180$ & M \\
\hline 651 & La Jolla kelp & 850,650 & $310,290,190,150$ & 510,480 & $\mathrm{~N}$ \\
\hline 413 & Catalina kelp & Uncut & $310,290,210,175,150$ & 550,520 & $\mathrm{O}$ \\
\hline 124 & Catalina water & Uncut & $390,310,290,190,150$ & 550,520 & $\mathrm{P}$ \\
\hline 637 & Pt. Loma kelp & Uncut & $500,290,190,125,100$ & $550,520,125$ & Q \\
\hline
\end{tabular}


Table 1 continued

\begin{tabular}{llllll}
\hline Stock number & Location & ScaI & HaeIII & MspI & Restriction pattern \\
\hline $\mathbf{4 8 1}$ & Pt. Loma kelp & Uncut & $650,290,250,190$ & $800,450,125$ & $\mathrm{R}$ \\
\hline
\end{tabular}

Bold numbers indicate sequenced Isolates from each group

Table 2 BLASTn results of 16S rDNA sequences from each RFLP pattern

\begin{tabular}{|c|c|c|c|c|c|c|c|}
\hline $\begin{array}{l}\text { Sequenced stock } \\
\text { number }\end{array}$ & $\begin{array}{l}\text { RFLP } \\
\text { pattern }\end{array}$ & $\begin{array}{l}\text { number of } \\
\text { isolates }\end{array}$ & $\begin{array}{l}\text { Percent found } \\
\text { in kelp }\end{array}$ & Class & Family & $\begin{array}{l}\text { Hypothesized } \\
\text { classification }\end{array}$ & $\begin{array}{l}\text { Accession } \\
\text { number }\end{array}$ \\
\hline ED401 & A & 31 & 45.16 & $\begin{array}{l}\text { Gamma } \\
\text { proteobacteria }\end{array}$ & Vibrionaceae & Vibrio sp. & KF442438 \\
\hline ED478 & B & 9 & 66.6 & $\begin{array}{l}\text { Gamma } \\
\text { proteobacteria }\end{array}$ & Vibrionaceae & Vibrio sp. & KF442439 \\
\hline ED121 & $\mathrm{C}$ & 9 & 33.3 & $\begin{array}{l}\text { Gamma } \\
\text { proteobacteria }\end{array}$ & Vibrionaceae & Vibrio sp. & KF442440 \\
\hline ED575 & $\mathrm{D}$ & 8 & 100 & $\begin{array}{l}\text { Alpha } \\
\text { proteobacteria }\end{array}$ & Rhodobacteraceae & Ruegeria sp. & KF442441 \\
\hline ED583 & $\mathrm{E}$ & 8 & 50 & $\begin{array}{l}\text { Gamma } \\
\text { proteobacteria }\end{array}$ & Alteromonadaceae & $\begin{array}{l}\text { Pseudoalteromonas } \\
\text { sp. }\end{array}$ & KF442442 \\
\hline ED588 & $\mathrm{F}$ & 6 & 83.3 & $\begin{array}{l}\text { Gamma } \\
\text { proteobacteria }\end{array}$ & Alteromonadaceae & $\begin{array}{l}\text { Pseudoalteromonas } \\
\text { sp. }\end{array}$ & KF442443 \\
\hline ED187 & G & 5 & 0 & $\begin{array}{l}\text { Gamma } \\
\text { proteobacteria }\end{array}$ & Vibrionaceae & Photobacterium sp. & KF442444 \\
\hline ED641 & $\mathrm{H}$ & 5 & 80 & $\begin{array}{l}\text { Gamma } \\
\text { proteobacteria }\end{array}$ & Vibrionaceae & Vibrio sp. & KF442445 \\
\hline ED329 & I & 3 & 66.6 & $\begin{array}{l}\text { Gamma } \\
\text { proteobacteria }\end{array}$ & Shewanellaceae & Shewanella sp. & KF442446 \\
\hline ED248 & $\mathrm{J}$ & 2 & 50 & $\begin{array}{l}\text { Gamma } \\
\text { proteobacteria }\end{array}$ & Alteromonadaceae & $\begin{array}{l}\text { Pseudoalteromonas } \\
\text { sp. }\end{array}$ & KF442447 \\
\hline ED589 & $\mathrm{K}$ & 2 & 100 & $\begin{array}{l}\text { Gamma } \\
\text { proteobacteria }\end{array}$ & Alteromonadaceae & $\begin{array}{l}\text { Pseudoalteromonas } \\
\text { sp. }\end{array}$ & KF442448 \\
\hline ED486 & $\mathrm{L}$ & 2 & 100 & $\begin{array}{l}\text { Gamma } \\
\text { proteobacteria }\end{array}$ & Alteromonadaceae & Alteromonas sp. & KF442449 \\
\hline ED142 & M & 2 & 100 & $\begin{array}{l}\text { Gamma } \\
\text { proteobacteria }\end{array}$ & Alteromonadaceae & $\begin{array}{l}\text { Pseudoalteromonas } \\
\text { sp. }\end{array}$ & KF442450 \\
\hline ED651 & $\mathrm{N}$ & 1 & 100 & $\begin{array}{l}\text { Gamma } \\
\text { proteobacteria }\end{array}$ & Vibrionaceae & Vibrio sp. & KF442451 \\
\hline ED413 & $\mathrm{O}$ & 1 & 100 & $\begin{array}{l}\text { Gamma } \\
\text { proteobacteria }\end{array}$ & Vibrionaceae & Vibrio sp. & KF442452 \\
\hline ED124 & $\mathrm{P}$ & 1 & 0 & $\begin{array}{l}\text { Gamma } \\
\text { proteobacteria }\end{array}$ & Vibrionaceae & Vibrio sp. & KF442453 \\
\hline ED637 & Q & 1 & 100 & $\begin{array}{l}\text { Gamma } \\
\text { proteobacteria }\end{array}$ & Alteromonadaceae & $\begin{array}{l}\text { Pseudoalteromonas } \\
\text { sp. }\end{array}$ & KF442454 \\
\hline ED481 & $\mathrm{R}$ & 1 & 100 & Flavobacteria & Flavobacteriaceae & Psychroserpens sp. & KF442455 \\
\hline Total & 18 & 97 & & 3 & 5 & 18 & \\
\hline
\end{tabular}

Loma compared to Catalina $\left[\overline{\mathrm{x}}_{\mathrm{PtLoma}}=12.210( \pm 0.015){ }^{\circ} \mathrm{C}\right.$, $\left.\overline{\mathrm{x}}_{\text {Catalina }}=15.167( \pm 0.003){ }^{\circ} \mathrm{C}\right](p<0.001)$. In addition, $\mathrm{pH}$ was higher in Catalina at surface $\left[\overline{\mathrm{x}}_{\mathrm{PtLoma}}=8.073\right.$ $\left.( \pm 0.055), \quad \overline{\mathrm{x}}_{\text {Catalina }}=8.847( \pm 0.009)\right] \quad(p=0.004)$ and benthos $\left[\overline{\mathrm{x}}_{\text {PtLoma }}=8.397 \quad( \pm 0.003), \overline{\mathrm{x}}_{\text {Catalina }}=8.783\right.$ $( \pm 0.007)](p<0.001)$. Furthermore, chlorophyll-a was higher at the surface of Pt. Loma $\left[\overline{\mathrm{x}}_{\text {PtLoma }}=1.347\right.$ $\left.( \pm 0.015) \mathrm{mg} / \mathrm{l}, \quad \overline{\mathrm{x}}_{\text {Catalina }}=1.253 \quad( \pm 0.018) \mathrm{mg} / \mathrm{l}\right] \quad(p=$ $0.016)$, while dissolved oxygen was lower $\left[\overline{\mathrm{x}}_{\text {PtLoma }}=\right.$ $\left.6.123( \pm 0.023) \mathrm{mg} / \mathrm{l}, \overline{\mathrm{x}}_{\text {Catalina }}=8.880( \pm 0.010) \mathrm{mg} / \mathrm{L}\right]$ $(p<0.001)$ and salinity was higher $\left[\overline{\mathrm{x}}_{\mathrm{PtLoma}}=45.361\right.$ $\left.( \pm 0.026) \mathrm{ppt}, \overline{\mathrm{x}}_{\text {Catalina }}=41.770( \pm 0.027) \mathrm{ppt}\right](p<0.001)$ 


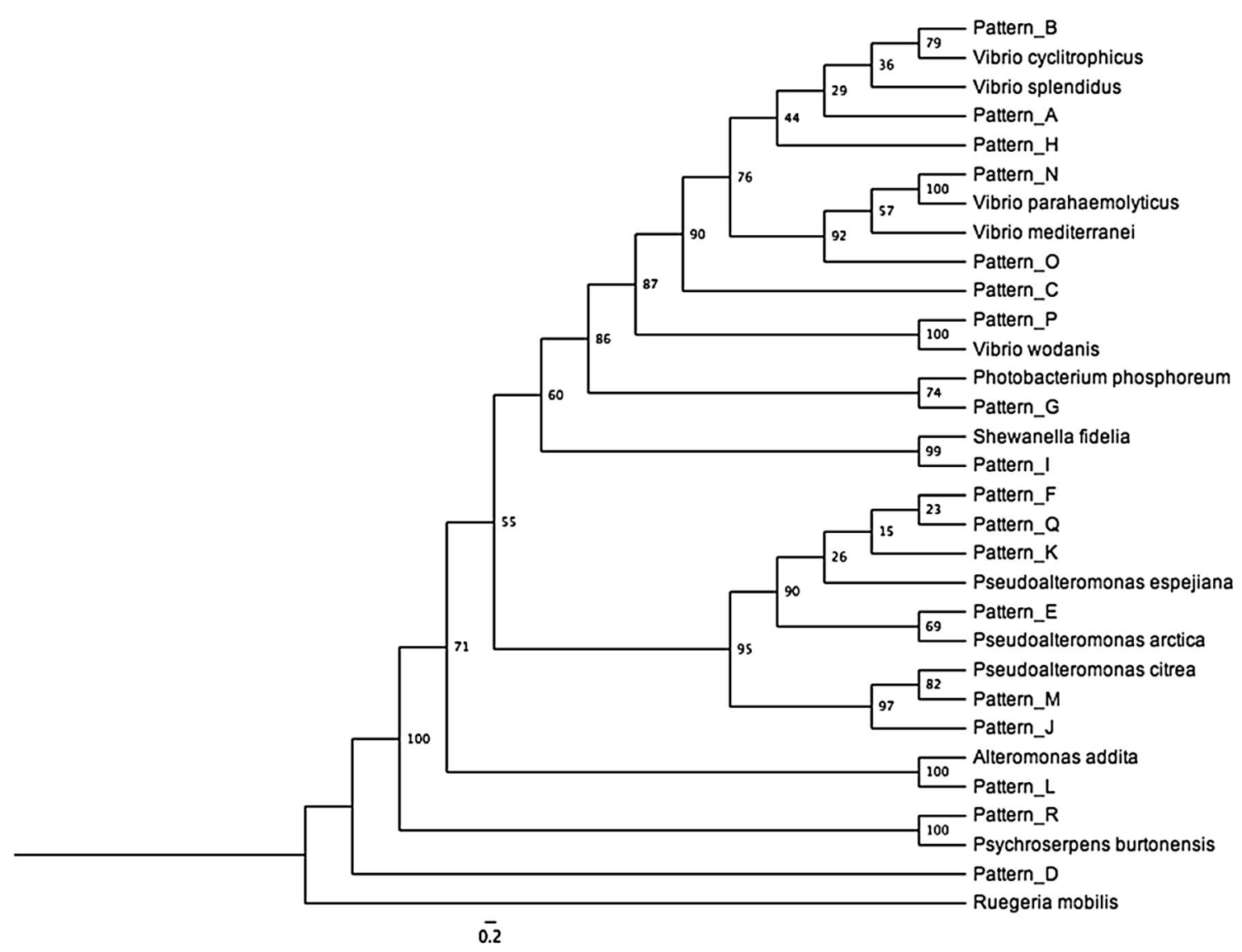

Fig. 2 Phylogenetic tree of 16S rDNA sequences created from a MUSCLE alignment using RAxML. Bootstrap values (100 iterations) are indicated at the nodes. The scale bar indicates 0.2 mutations per site

at the benthos of Pt. Loma compared to Catalina (Table 3).

For all kelp forest bacterial isolates, the lowest concentrations of copper in the media resulted in a slight decrease in optical density. After the decrease, bacterial growth was maintained until a steep drop was observed in the graph of absorbance versus copper concentration suggesting the copper concentration had reached an inhibitory level (Fig. 3a-c). The $\mathrm{IC}_{50}$ data for Pt. Loma isolates $(n=10)$ showed higher copper tolerance with the majority of growth inhibition occurring between 200 and 250 ppm Cu (Fig. 3a). The bacterial isolates from La Jolla $(n=10)$ showed growth inhibition occurring between 150 and 275 ppm Cu (Fig. 1b). Catalina isolates $(n=9)$ showed growth inhibition occurring between 100 and $225 \mathrm{ppm}$ of $\mathrm{Cu}$ (Fig. 3c).

The minimal inhibitory concentration varied with location $\left(\mathrm{F}_{\mathrm{df}=2}=41.215\right.$ and $\left.p<0.001\right)$, sample type $\left(\mathrm{F}_{\mathrm{df}=1}=4.018\right.$ and $p=0.047)$ and there was an interaction between location and sample type $\left(\mathrm{F}_{\mathrm{df}=2}=11.394\right.$ and $\left.p<0.001\right)$ (Fig. 3d). The minimal inhibitory concentration was highest at Pt. Loma and the kelp associated bacteria had a higher $\mathrm{IC}_{50}$ than the water associated bacteria. The second highest $\mathrm{IC}_{50}$ values were found at La Jolla, but the water associated bacteria had higher $\mathrm{IC}_{50}$ than the kelp associated bacteria. Catalina showed the lowest $\mathrm{IC}_{50}$ and once again the kelp associated bacteria had higher $\mathrm{IC}_{50}$ than the water associated bacteria. Trends of the copper tolerance of the community of bacteria were observed in specific bacterial species where five isolates similar to $V$. cyclitrophicus from $M$. pyrifera and seawater showed different values for copper tolerance across the three geographic locations (Fig. 3e). The copper tolerance of isolates was negatively correlated with distance from human urbanization and marinas.

\section{Discussion}

High levels of urbanization, boating and the resultant copper introduced into the water column from antifouling paint may be capable of affecting the structure and function of bacterial populations in kelp forests. Bacterial isolates from Pt. Loma, the location with highest measured copper levels, were able to tolerate copper concentrations significantly higher than bacteria isolated from La Jolla and Catalina, locations with less human activity (Fig. 3d). We showed that the input of transient metals causes a 
Table 3 Water quality measurements taken with a MANTA-2 multiprobe

\begin{tabular}{crrrrrrrr}
\hline & Depth $(\mathrm{m})$ & Temp $\left({ }^{\circ} \mathrm{C}\right)$ & Salinity $(\mathrm{ppt})$ & $\mathrm{pH}$ & \multicolumn{2}{l}{$\begin{array}{l}\text { Chlorophyll-a (mg/ } \\
1)\end{array}$} & $\begin{array}{l}\text { Dissolved } \\
\text { oxygen } \\
(\% \text { sat })\end{array}$ & $\begin{array}{l}\text { Dissolved } \\
\text { oxygen } \\
(\mathrm{mg} / \mathrm{l})\end{array}$ \\
\hline Pt. & $0.607 \pm 0.047$ & $16.807 \pm 0.039$ & $40.308 \pm 0.039$ & $8.073 \pm 0.055$ & $1.347 \pm 0.015$ & $118.067 \pm 2.034$ & $9.367 \pm 0.153$ \\
Loma & $13.950 \pm 0.183$ & $12.210 \pm 0.015$ & $45.361 \pm 0.026$ & $8.397 \pm 0.003$ & $3.853 \pm 0.116$ & $70.267 \pm 0.260$ & $6.123 \pm 0.023$ \\
Catalina & $0.230 \pm 0.006$ & $16.593 \pm 0.009$ & $40.205 \pm 0.010$ & $8.847 \pm 0.009$ & $1.253 \pm 0.018$ & $115.000 \pm 0.808$ & $9.177 \pm 0.064$ \\
& $11.490 \pm 0.074$ & $15.167 \pm 0.003$ & $41.770 \pm 0.027$ & $8.783 \pm 0.007$ & $3.973 \pm 0.766$ & $108.233 \pm 0.167$ & $8.880 \pm 0.010$ \\
\hline
\end{tabular}

Data was logged at surface and benthos depths for each water collection site; Pt. Loma and Catalina Island, California kelp forests. Values displayed are the mean for three replicate measurements, with standard error of the mean (SEM)
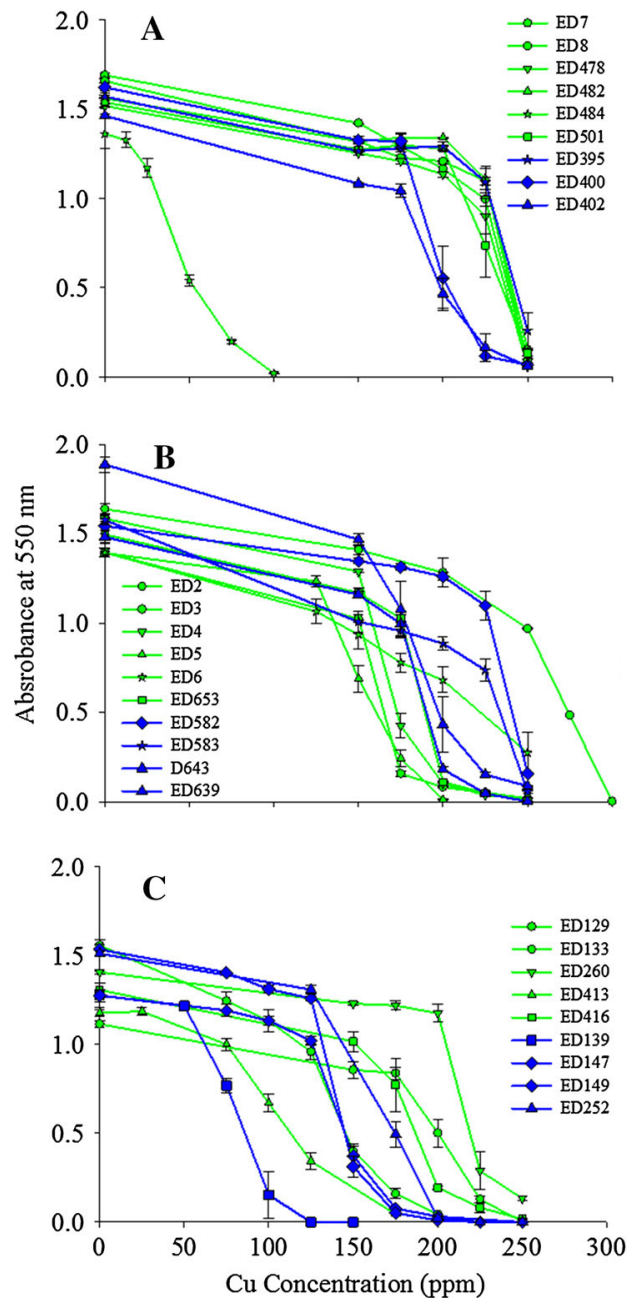

Fig. 3 a The concentration of copper that inhibited bacterial growth in isolates from Pt. Loma was found to be above $200 \mathrm{ppm}$ in all but one sample. b The concentration of copper that inhibited bacterial growth in isolates from La Jolla was found to range from 150 to $275 \mathrm{ppm}$. c The concentration of copper that inhibited bacterial growth in isolates from Catalina was found to range from 100 to $225 \mathrm{ppm}$. d Mean copper inhibitory growth concentrations of $M$. pyrifera (green) and seawater (blue) associated bacterial isolates across three geographic locations. e The copper MIC assay data for
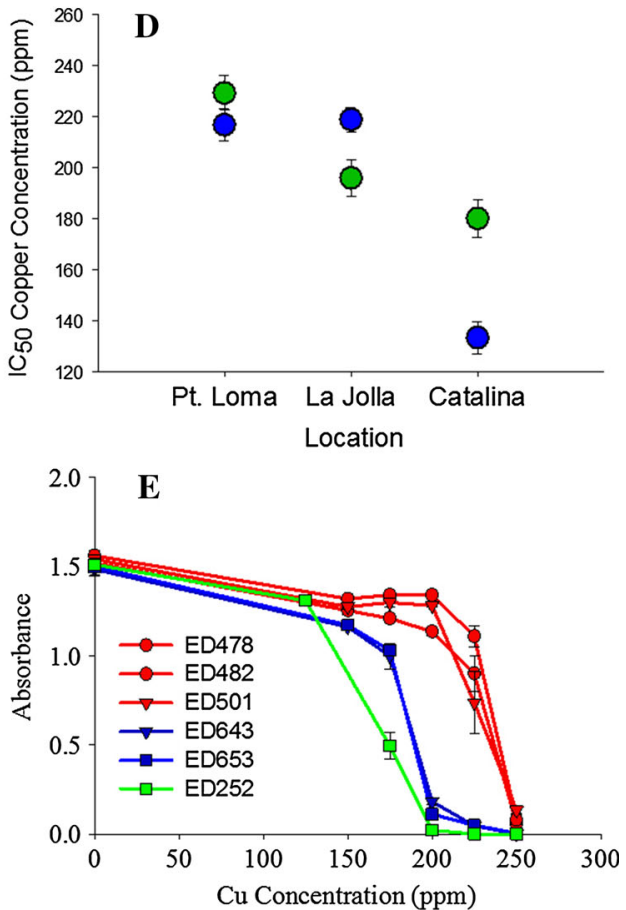

bacterial species classified as similar to $V$. cyclitrophicus by $16 \mathrm{~S}$ rDNA isolated from Pt. Loma (red), La Jolla (blue), and Catalina (green). The isolates from Pt. Loma were the most resistant to copper while the one collected from Catalina was the least copper tolerant. Isolate numbers used in the MIC are from: Pattern A-ED400, ED3, ED4, ED5, ED582, ED147,ED149, Pattern B-D8, ED478, ED482, ED395, ED653, ED643, Pattern C-ED129, Pattern E-ED583, ED484, Pattern G-ED639, Pattern H-ED2, ED133, Pattern IED416, ED139, Pattern O-ED413 (Color figure online) 
physiological change (tolerance to copper) in the bacteria and the ramifications of these changes on the kelp health will be the goals of future research. Because a range of bacterial species were tested, we show that the development of copper tolerance was location rather than species specific, suggesting that bacteria that lived in a higher copper habitat develop the ability to withstand higher levels of copper.

In addition to the positive correlation between copper pollution and bacterial copper resistance across different species, we observed that strains of a single species, similar to $V$. cyclitrophicus, had higher copper tolerance from Catalina to La Jolla, and the greatest tolerance was found in isolates from Pt. Loma (Fig. 3e). The results of increased copper tolerance in bacteria from areas with higher levels of copper are consistent with findings that Enterobacteriaceae strains from marine sediments and seawater have copper resistance directly influenced by pollution (Altug and Balkis 2009). These results suggest that some marine bacteria are able to increase their tolerance to higher levels of copper when living in locations with elevated inputs of transient metals.

Copper in the oceanic water column is reported to be 0 . $225 \mathrm{ppb}$ (Blossom 2007) and levels in San Diego Bay was $\sim 8$ ppb (Johnson and Gonzalez 2005; Neira et al. 2011), and we found that the copper in the kelp at Pt. Loma and Catalina was high, a mean 6.21 and 4.5 ppm respectively, showing the kelp is concentrating the copper from the water column. The amount of copper in an organism depends on the diffusion across tissue boundaries and the amount to which it is retained in the organism (Neira et al. 2011). The switch in higher copper tolerance in the microbes isolated from the water column compared to the kelp at La Jolla, suggests that at this location the copper may be returning to solution at a faster rate than at Pt. Loma or Catalina. Although the concentrations of copper at which bacterial growth was inhibited for the majority of isolates were above the levels found in M. pyrifera from Pt. Loma and Catalina, our results suggests that the transient metals in the environment are correlated with a phenotypic change in the bacteria. In addition previous studies have shown that the brown macroalgae Ascophyllum sp. and Laminaria japonica can accumulate 80.06 and $100.03 \mathrm{ppt}$ of $\mathrm{Cu}$ respectively (Mehta and Gaur 2005), which is 1000 fold greater than the concentrations shown to inhibit growth of the bacterial isolates (Fig. 3). If the antifoulant paint used on boats in the San Diego Bay and other marinas worldwide continue to contribute copper to the water, in addition to other anthropogenic inputs, the algae in adjacent kelp forests such as Pt. Loma are capable of accumulating lethal concentrations of copper for even the most resistant bacterial isolate and these changes in epibiosis may affect kelp health.
A distinct bacterial communities associated with giant kelp were identified. Of the 18 RFLP patterns observed, ten were found in a particular location and ten are specific to either kelp or seawater. These results support the hypothesis that kelp forms a distinct microhabitat that selects for certain species of bacteria that are rare in the surrounding water and we are currently investigating the genotypes of these species to identify why they a specific to either kelp or water column. Vibrio species were the dominant organism cultured from the kelp and surrounding water (Table 1). These gram negative, motile rod-shaped species are ubiquitous in marine environments and are found in especially high amounts on eukaryotic host organisms, such as coral and algae (Thompson et al. 2004). This is consistent with our findings as bacterial species from the Vibrio genus were the most widespread in terms of location as well as the most abundant. Pseudoalteromonas is another well-represented marine Gamma proteobacteria and representatives from this genus have been isolated from both brown and green algae as well as seawater (Egan et al. 2008; Sawabe et al. 1998; Staufenberger et al. 2008). Shewanella species have previously been isolated from both seawater and marine organisms and also have the ability to digest agar (Ivanova et al. 2001, 2004). While some species were isolated from both sample types across all sampling sites, many were exclusive to geographical location. Catalina and Pt. Loma, the sites with the least and greatest anthropogenic effects, respectively, have more species that appear to be specific to the locations than La Jolla. Our current research is investigating the noncultured organisms associated with the kelp using next generation DNA sequencing of metagenomes, which will provide a more complete picture of the kelp microbiome. By culturing bacteria we are able to conduct phenotypic analysis, which are not possible with metagenomics. In addition, we are sequencing the genomes of the cultured bacteria to understand the underpinnings of the phenotypic differences in copper tolerance of the bacteria.

The results of this study suggest that marine microbes are subject to selection at the species level across different geographical locations with varying anthropogenic and industrial influences. Furthermore, kelp appears to select for certain species of bacteria, creating a diverse and distinct microbiome on the algal surface. Kelp forests near industrial areas appear to be decreasing in cover and to date, have failed to recover to pre-1950 levels (Coleman et al. 2008; Connell et al. 2008; Foster and Schiel 2010). The consequences of an altered microbial community could have dramatic effects on the health of the ecosystem and warrants further investigation.

Acknowledgments Dinsdale is supported by NSF Division of Undergraduate Education \#1323809 and Division of Microbial Biology 
\#1330800, and FIPSE-CAPES USA-Brazil Higher Education Consortia Program. Nascimento and Magalhães are supported by CAPESFIPSE. Dutilh is supported by the Dutch Science foundation (NWO) Veni Grant 016.111.075.

Conflict of interest The authors declare that they have no conflict of interest.

Open Access This article is distributed under the terms of the Creative Commons Attribution 4.0 International License (http:// creativecommons.org/licenses/by/4.0/), which permits unrestricted use, distribution, and reproduction in any medium, provided you give appropriate credit to the original author(s) and the source, provide a link to the Creative Commons license, and indicate if changes were made.

\section{References}

Altug G, Balkis N (2009) Levels of some toxic elements and frequency of bacterial heavy metal resistance in sediment and sea water. Environ Monit Assess 149:61-69. doi:10.1007/ s10661-008-0183-z

Andrade LR, Salgado LT, Farina M et al (2004) Ultrastructure of acidic polysaccharides from the cell walls of brown algae. J Struct Biol 145:216-225. doi:10.1016/j.jsb.2003.11.011

Andrade LR, Leal RN, Noseda $M$ et al (2010) Brown algae overproduce cell wall polysaccharides as a protection mechanism against the heavy metal toxicity. Mar Pollut Bull 60:1482-1488. doi:10.1016/j.marpolbul.2010.05.004

Armstrong E, Yan L, Boyd KG et al (2001) The symbiotic role of marine microbes on living surfaces. Water 461:37-40

Biggs TW, D'Anna H (2012) Rapid increase in copper concentrations in a new marina, San Diego Bay. Mar Pollut Bull 64:627-635. doi:10.1016/j.marpolbul.2011.12.006

Blight AJ, Thompson RC (2008) Epibiont species richness varies between holdfasts of a northern and a southerly distributed kelp species. J Mar Biol Assoc UK 88:469-475. doi:10.1017/ S0025315408000994

Blossom N (2007) Copper in the Ocean Environment. Am Chemet Corp 1-8

Burke C, Kjelleberg S, Thomas T (2009) Selective extraction of bacterial DNA from the surfaces of macroalgae. Appl Environ Microbiol 75:252-256. doi:10.1128/AEM.01630-08

Coleman MA, Kelaher BP, Steinberg PD, Millar AJK (2008) Absence of a large brown macroalgae on urbanized rocky reefs around Sydney, Australia, and evidence for historical decline 1. J Phycol 44:897-901. doi:10.1111/j.1529-8817.2008.00541.x

Connell S, Russell B, Turner D et al (2008) Recovering a lost baseline: missing kelp forests from a metropolitan coast. Mar Ecol Prog Ser 360:63-72. doi:10.3354/meps07526

Davis TA, Volesky B, Mucci A (2003) A review of the biochemistry of heavy metal biosorption by brown algae. Water Res 37:4311-4330. doi:10.1016/S0043-1354(03)00293-8

Dobalian L, Arias CM (2005) Total maximum daily load for dissolved copper in Shelter Island Yacht Basin. San Diego Regional Water Quality Board, Executive Officer's Report, 16 pp

Edgar RC (2004) MUSCLE: multiple sequence alignment with high accuracy and high throughput. Nucleic Acids Res 32:1792-1797. doi: $10.1093 /$ nar/gkh340

Egan S, Thomas T, Kjelleberg S (2008) Unlocking the diversity and biotechnological potential of marine surface associated microbial communities. Curr Opin Microbiol 11:219-225. doi:10.1016/j.mib.2008.04.001

Foster MS, Schiel DR (2010) Loss of predators and the collapse of southern California kelp forests (?): alternatives, explanations and generalizations. J Exp Mar Bio Ecol 393:59-70. doi:10. 1016/j.jembe.2010.07.002

Goecke F, Labes A, Wiese J, Imhoff J (2010) Chemical interactions between marine macroalgae and bacteria. Mar Ecol Prog Ser 409:267-299. doi:10.3354/meps08607

Ivanova EP, Sawabe T, Gorshkova NM et al (2001) Shewanella japonica sp. nov. Int J Syst Evol Microbiol 51:1027-1033

Ivanova EP, Gorshkova NM, Bowman JP et al (2004) Shewanella pacifica sp. nov., a polyunsaturated fatty acid-producing bacterium isolated from sea water. Int J Syst Evol Microbiol 54:1083-1087. doi:10.1099/ijs.0.02993-0

Lachnit T, Meske D, Wahl M et al (2011) Epibacterial community patterns on marine macroalgae are host-specific but temporally variable. Environ Microbiol 13:655-665. doi:10.1111/j.14622920.2010.02371.x

Mehta SK, Gaur JP (2005) Use of algae for removing heavy metal ions from wastewater: progress and prospects. Crit Rev Biotechnol 25:113-152. doi:10.1080/07388550500248571

Moffett JW, Brand LE, Croot PL et al (2012) Cu speciation and cyanobacterial distribution in harbors subject to anthropogenic $\mathrm{Cu}$ inputs. Limnol Oceanogr 42:789-799

Neira C, Mendoza G, Levin LA, Zirino A, Delgadillo-Hinojosa F, Porrachia M, Deheyn DD (2011) Macrobenthic community response to copper in Shelter Island Yacht Basin, San Diego Bay, California. Mar Poll Bull 62:701-717

Santelices B (2007) The discovery of kelp forests in deep-water habitats of tropical regions. Proc Natl Acad Sci USA 104:19163-19164. doi:10.1073/pnas.0708963104

Sawabe T, Makino H, Tatsumi M et al (1998) Pseudoalteromonas bacteriolytica sp. nov., a marine bacterium that is the causative agent of red spot disease of Laminaria japonica. Int J Syst Bacteriol 48:769-774

Schiff K, Brown J, Diehl D (2006) Extent and magnitude of copper contamination in Marinas of the San Diego Region, California. Water Res 54:322-328

Stamatakis A (2014) RAxML version 8: a tool for phylogenetic analysis and post-analysis of large phylogenies. Bioinformatics 30:1312-1313. doi:10.1093/bioinformatics/btu033

Staufenberger T, Thiel V, Wiese J, Imhoff JF (2008) Phylogenetic analysis of bacteria associated with Laminaria saccharina. FEMS Microbiol Ecol 64:65-77. doi:10.1111/j.1574-6941. 2008.00445.x

Stuart RK, Dupont CL, Johnson DA et al (2009) Coastal strains of marine Synechococcus species exhibit increased tolerance to copper shock and a distinctive transcriptional response relative to those of open-ocean strains. Appl Environ Microbiol 75:5047-5057. doi:10.1128/AEM.00271-09

Thompson FL, Iida T, Swings J (2004) Biodiversity of Vibrios. Microbiol Mol Biol Rev 68:403-431. doi:10.1128/MMBR.68.3. 403

Warnau M, Ledent G, Temaral A, Jangouxr M (1995) Experimental cadmium contamination of the echinoid Paracentrotus lividus: influence of exposure mode and distribution of the metal in the organism. Mar Ecol Prog Ser 116:117-124

Johnson LT, Gonzalez JA (2005) Nontoxic antifouling? Demonstrating a solution to copper boat bottom paint pollution. In Proceedings of the 14th Biennial Coastal Zone Conference 